\author{
The technique that works on one patient may not \\ suit another, and the tip that saves time in the \\ hands of one dentist may be useless in the hands \\ of another.
}

\title{
One size fits all
}

One of the most popular sessions at the recent BDA Conference in Belfast was the 'Tips and Techniques' session where dentists swapped ideas and tips with each other. It is hardly surprising that this is a popular session, partly because we are all looking for ideas to make our work easier and more effective, but also because there is something very appealing about the easy answer or the 'cookbook' approach that suggests there is a simple recipe that works for every situation.

As we all know the easy answer is not always the best, and tips and techniques only work when you have a solid understanding of the subject. This is because, despite the attractive slogan, one size never does fit all. The technique that works on one patient may not suit another, and the tip that saves time in the hands of one dentist may be useless in the hands of another. Thankfully, at least in clinical matters, the fact that all dentists have a thorough grounding in clinical dentistry enables them to use judgement in applying the various ideas suggested in a 'tips and techniques' session.

Alas, the same is not true when it comes to dental business matters. Many dentists do not have an understanding of business principles and, without that basic solid foundation of knowledge they can often believe that an idea suggested by a colleague or business consultant will always work in their practice.

Let me provide two different examples to illustrate what I mean. The first example involves the suggestion that a bonus system will motivate staff. This may work, but before introducing a bonus system you need to be very clear on the business objectives of the practice, the financial objectives and the financial strategy. You should also have clear financial models to predict the outcome of applying a bonus system along with an understanding of the financial performance of the practice so far and a fairly clear idea of what you think will happen to both dentistry and your practice in the near future. All this is quite clear when you have a good understanding of business principles, making the decision as to whether to introduce a bonus system a reasoned and considered one. 